Review article

\title{
Impact of COVID-19 pandemic on audiology practice: A scoping review
}

\author{
Komal Aggarwal ${ }^{\mathrm{a}}$, Dhanshree R. Gunjawate ${ }^{\mathrm{b}}$, Krishna Yerraguntla ${ }^{\mathrm{a}}$, Rohit Ravi ${ }^{\mathrm{b},{ }^{*}}$ \\ ${ }^{a}$ Department of Speech and Hearing, Manipal College of Health Professionals, Manipal Academy of Higher Education, Manipal, Karnataka, India \\ ${ }^{\mathrm{b}}$ Department of Audiology and Speech Language Pathology, Kasturba Medical College, Mangalore Manipal Academy of Higher Education, Manipal, Karnataka, India
}

\section{A R T I C L E I N F O}

\section{Keywords:}

COVID-19

Audiology

Practice

Pandemic

Audiologists

\begin{abstract}
A B S T R A C T
Background: COVID-19 has significantly impacted the professional practice of health care professionals including audiological practice. Audiologists had to adopt to changes and modify their practice to deal with the pandemic. Reasonable published work has been seen since the onset of the pandemic, depicting impact on the audiology practice across the globe in different practice settings and contexts. The present scoping review was carried out to explore the impact of COVID-19 pandemic on audiology practice and the challenges faced.

Material \& methods: A literature search was carried out to identify the studies exploring the impact of COVID-19 pandemic on audiology practice. Three electronic databases namely Scopus, PubMed/Medline, and Cochrane Library were searched using keywords. After title and abstract screening, suitable studies were identified. Results: Of the total 172, nine studies focusing on impact of COVID-19 pandemic on audiology practice COVID19, published between January 2020 to June 2021 were included. Overall, the COVID-19 pandemic and the subsequent restrictions, caused changes in routine clinical practices in audiology. These changes were with respect to stringent infection control measures, mode of service delivery, changes in routines with reduced services or prioritizing services. Challenges included issues with remote services, experience, and support. Conclusion: The present review reveals the changes in audiology practice during COVID -19 and challenges encountered by audiologists. These findings would help in better planning of audiology practice in the post pandemic world.
\end{abstract}

\section{Introduction}

Pandemics are simultaneous global transmission of infectious diseases affecting significantly large numbers of people leading to high morbidity and mortality, social, political, and economic disruption. ${ }^{1}$ These pandemics have several short-term as well as long-term implications on the health care systems, especially on the healthcare professionals. ${ }^{2,3}$ The coronavirus disease-19 (also called as COVID-19) emerged in Wuhan, China, and eventually rapidly spread across the globe. ${ }^{4}$ The subsequent lockdowns and stay-at-home orders have led to several disruptions in availability and access to healthcare services. A recent systematic review reported a drastic reduction to almost one-third in the utilization of healthcare services, especially among persons with less severe illnesses. ${ }^{5}$

As restrictions were imposed as well as while they were being relaxed, healthcare services were either halted or reduced or shifted to online modality. These changes in service delivery were carried out to avoid the spread of infection and ensure patients are cared about. The healthcare professionals also had to adapt to what was termed as the "new normal" for infection control measures, hand hygiene, social distancing, and changes in routine clinical procedures to reduce or prevent risk of transmission. ${ }^{6,7}$

Audiological consultation usually take place in closed sound-treated rooms. Further, multiple face-to-face appointments are required for assessment, hearing aid trial, fitting, counselling, and troubleshooting. ${ }^{8,9}$ In audiology, the catastrophe of COVID-19 had a profound impact requiring modifications to existing practices as well as adaptations to the rapidly evolving situation. Professional bodies responded rapidly and provided guidelines and positional statements to help the audiologists adapt to the changing clinical scenario. ${ }^{10-13}$ Tele-practice had always been an integral part of clinical practice in audiology, even before the pandemic. ${ }^{14,15}$ However, the use of tele-practice got an added impetus during the pandemic.

Although the COVID-19 pandemic has limited the access and provision of healthcare services, it is equally important to continue delivering audiological services. Reasonable published work has been seen

\footnotetext{
* Corresponding author.

E-mail address: rohitravi94@gmail.com (R. Ravi).
} 

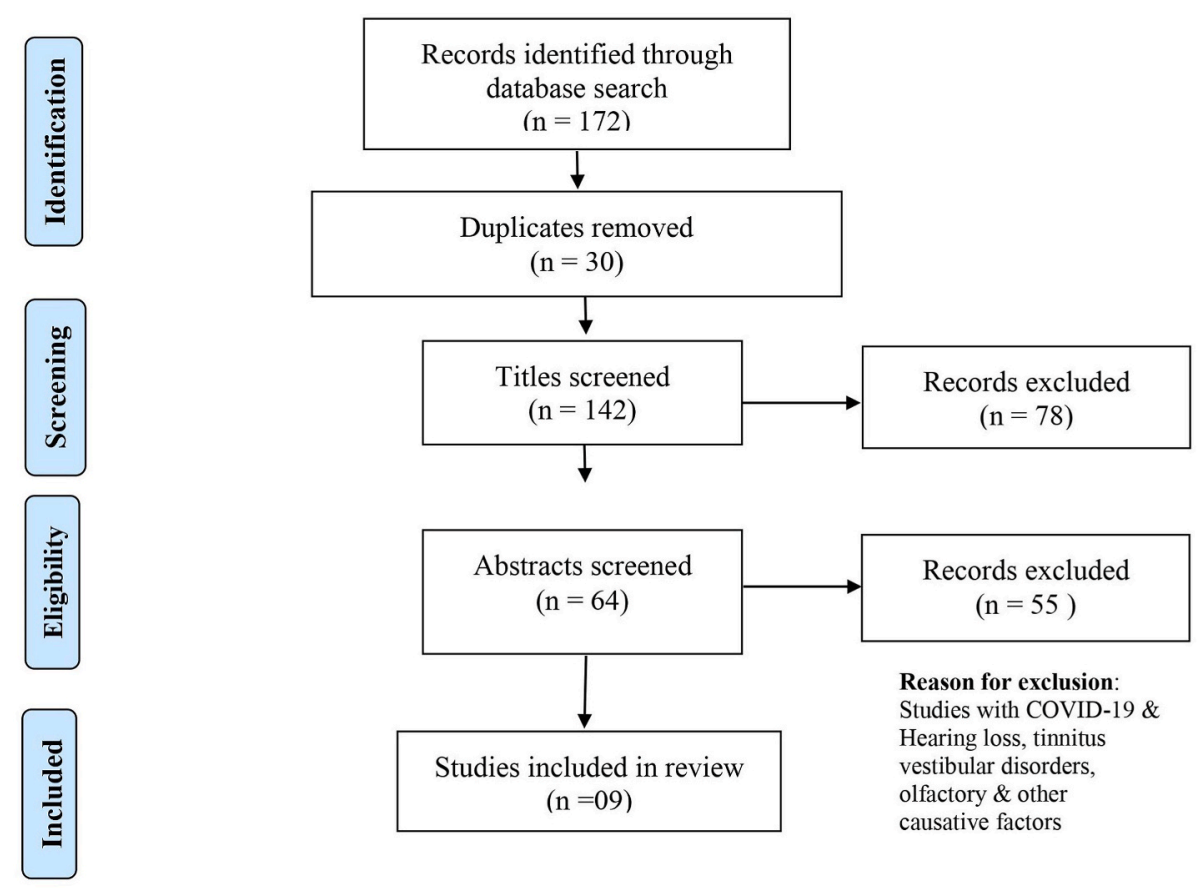

Figure 1. PRISMA flowchart explaining the steps followed in the review

since the onset of the pandemic, to study the impact on the audiology practice across the globe. It would be interesting to note the procedures adopted by audiologists to reduce risk of COVID-19 infection, and the impact and changes in professional practices due to the pandemic. Thus, the present scoping review was carried out to explore the impact of COVID-19 pandemic on audiology practice and the challenges faced.

\section{Material \& Methods}

The scoping review was carried out using the Preferred Reporting for Systematic Review and Meta-analysis statement-extension for scoping review (PRISMA-ScR statement). The secondary data study related to COVID-19 were permitted by Ethical committee.

\subsection{Literature search, inclusion/exclusion criteria}

The population, intervention/impact, and outcome (PIO) was framed. Studies carried out among audiologists (P), exploring the impact and challenges of COVID-19 pandemic (I) on their audiology practice (O) were included. Studies exploring the impact of COVID-19 on individuals with hearing loss, tinnitus, vestibular or other audiological issues were excluded. Only studies published in English language and published from January 2020 to June 2021 were included for this review.

\subsection{Search methods used for identification of the studies}

The search was conducted by two independent reviewers (KA, RR). The electronic databases Scopus, PubMed/Medline, and Cochrane Library were included for the review. The keywords 'audiology', 'audiologist', 'teleaudiology', 'COVID-19', 'coronavirus-19', were used in different combination using Boolean operators 'AND' and 'OR'.

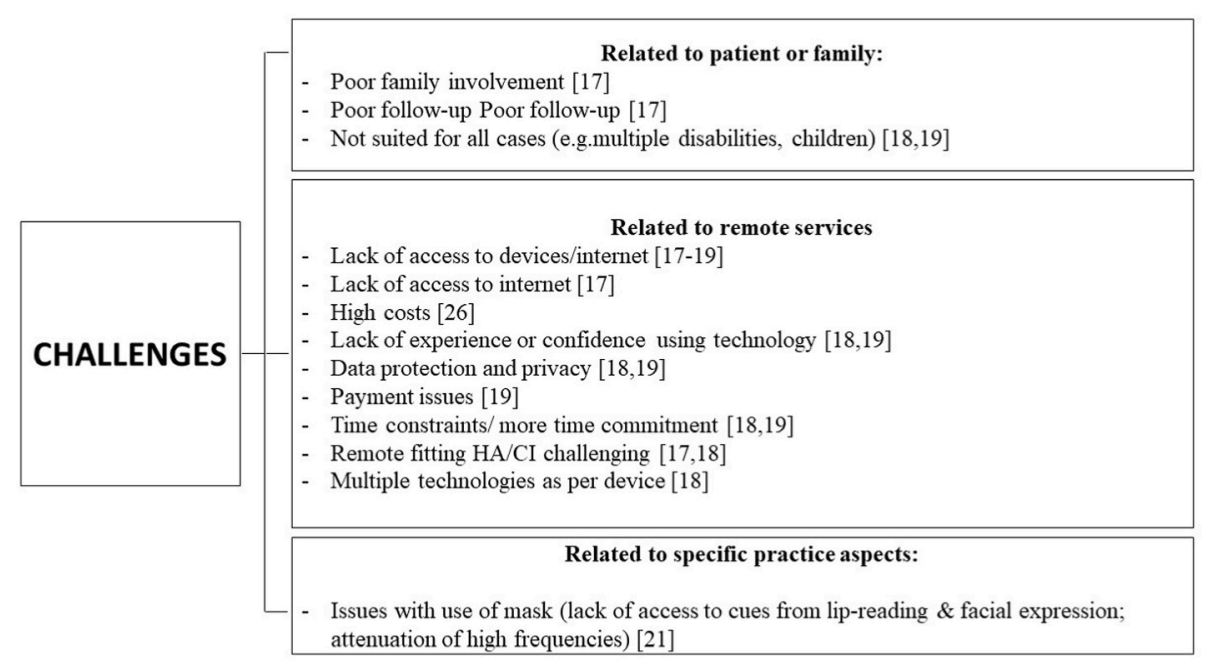

Figure 2. Challenges faced professional practice due to pandemic. 
Table 1

Characteristics of included studies.

\begin{tabular}{|c|c|c|c|}
\hline Author ID & Country & Study Design/sampling & Participants details \\
\hline $\begin{array}{c}\text { Gunjawate } \\
\text { et al. }{ }^{17}\end{array}$ & India & $\begin{array}{l}\text { Cross sectional study, using } \\
\text { convenient sampling }\end{array}$ & $\begin{array}{l}211 \text { audiologists \& speech-language } \\
\text { pathologists } \\
\text { Age: } 29.8 \pm 9.32 \text { years, } 21-69 \text { years } \\
\text { Experience: } 6.99 \pm 8.3 \text { years, } 1-40 \\
\text { years } \\
70 \% \text { females, } 28.6 \% \text { males, } 0.5 \% \text { no } \\
\text { revealed } \\
34.1 \% \text { Bachelors, } 57.8 \% \text { Masters, } \\
8.1 \% \text { Doctorate } \\
32.7 \% \text { private practice, } 31.3 \% \\
\text { hospital, } 36 \% \text { teaching and clinical } \\
\text { services }\end{array}$ \\
\hline $\begin{array}{l}\text { Manchaiah } \\
\text { et al. }^{18}\end{array}$ & $\begin{array}{l}\text { South Africa (International survey from } 44 \\
\text { countries, majority participants from five } \\
\text { countries Australia- 81, United States- } 60 \text {, } \\
\text { South Africa- 57, United Kingdom- 14, and } \\
\text { Canada-13) }\end{array}$ & Cross sectional study & $\begin{array}{l}337 \text { audiologists } \\
77 \% \text { females, } 23 \% \text { males } \\
\text { Age } 44.5 \text { years (22-81 years) } \\
\text { Experience } 18.5 \text { years ( } 0-53 \text { years). } \\
\text { Education level: } \\
\text { - On job training, no professional } \\
\text { degree-0.3\%; Certificate course- } \\
3.9 \% \text {, } \\
\text { - Bachelor's degree- } 19.6 \% \text {, } \\
\text { - Master's- } 38.3 \% \text {, } \\
\text { - Doctoral-38\% } \\
\text { Employment: } \\
\text { - Private practice- } 42.2 \% \text {, } \\
\text { - Private hospital or clinic- } 6.5 \% \text {, } \\
\text { Government hospital or clinic- } \\
23.1 \% \\
\text { - Others } 28.2 \%\end{array}$ \\
\hline $\begin{array}{l}\text { Parmar } \\
\text { et al. }^{19}\end{array}$ & UK & $\begin{array}{l}\text { Mixed-methods cross- } \\
\text { sectional survey, snowball } \\
\text { sampling }\end{array}$ & $\begin{array}{l}323 \text { hearing care professionals } \\
\text { ( } 67 \% \text { public sector; } 28 \% \text { private } \\
\text { sector) }\end{array}$ \\
\hline
\end{tabular}

Questionnaire details

23 items demographic details, knowledge and attitudes towards COVID-19, and practices related to infection control. Response - true/false statements, yes/no, multiple-choice questions, checkboxes, and short answer open-ended questions.

50 items demographic details, effects on the workplace, use of and attitude towards telehealth, mental health status. Response - Open ended questions \& structured closed set responses

Saunders
et $\mathrm{al}^{20}$

UK$$
\text { et al. }
$$

Survey conducted using social media \& personal emails, snowball sampling

120 audiologists

Work settings - 75\% paediatric care $57 \%$ conduct primary evaluations with adults, $58 \%$ hearing-aids fitting, $52.5 \%$ tinnitus care, $38.3 \%$ vestibular care.

\section{2 items}

Demographic information: Age, gender, regional location, educational qualifications, work setting, work role pre- and post- onset of COVID-19, duration of work, type of patients seen, time to commute to work Understanding about the term "Telehealth" Barriers to the delivery of telehealth Response - 58 closed-ended questions to be rated on 4-point Likert scale and 4 openended questions

28 items

- Practice patterns such as type of services provided (paediatric, adult evaluation, adult hearing-aids, tinnitus vestibular), location of practice, the number of daily appointments prior to COVID-19 restrictions.

- how each type of service provided has been managed during COVID-19 restrictions.

- decision-making \& triaging for remote care

- prior experience with remote care

- views about remote care \& its impacts on patient and service provision

Response - five-point rating scale or openended questions.

40 items across four sections

- demographic details, work, years of experience, gender, age, level of education, and population group they work with, children or adults.

- knowledge of the COVID-19 characteristics such (signs and symptoms, modes of transmission, incubation period, prevention methods).

- audiology practice during the COVID-19 pandemic, and precautions followed while providing services to patients

- audiologists' knowledge, attitude, and practice towards telehealth.

Response - yes/no or multiple-choices. NA 
Table 1 (continued)

\begin{tabular}{|c|c|c|c|c|}
\hline Author ID & Country & Study Design/sampling & Participants details & Questionnaire details \\
\hline Saki et al. ${ }^{23}$ & Iran & Commentary & NA & NA \\
\hline $\begin{array}{l}\text { Swanepoel } \\
\text { et al. }\end{array}$ & South Africa \& USA & Cover Story & NA & NA \\
\hline $\begin{array}{c}\text { Thai-Van } \\
\text { et al. }\end{array}$ & France & $\begin{array}{l}\text { Best practice } \\
\text { recommendations }\end{array}$ & NA & NA \\
\hline
\end{tabular}

NA- Not applicable.

\subsection{Screening of studies}

The hits from all the search engines were compiled together and the duplicate studies were eliminated. Two researchers independently screened the titles followed by the abstract for suitability of inclusion. The full-length of the shortlisted abstracts were then retrieved. Any discrepancies were resolved through discussion to arrive at a consensus. Data extraction was conducted from the full text of the hence shortlisted articles.

\subsection{Data extraction and management}

A data extraction sheet was prepared based on the discussion among all the authors. The extraction sheet included author id, country, study design, participant details, objective of the study, questionnaire/tool used, changes in professional practice due to COVID-19 pandemic and challenges/limitations faced.

\section{Results}

The total hits across all the databases were 172 and 30 duplicates were removed. 142 titles were screened to shortlist 64 abstracts for screening. Upon screening, 64 abstracts, 9 studies were included in the final review. Fig. 1 illustrates the Preferred Reporting Items for Systematic Review and Meta-Analyses (PRISMA) chart of the procedure followed to screen and shortlist the articles (Fig. 2).

\subsection{Summary of included studies}

The nine included studies in the review were from different countries, two from United Kingdom, one each from Jordan and Arab countries, India, France, South Africa, United States of America and Iran. One study was an international survey carried out by a group of authors from South Africa including participants from 44 countries, with most participants from five countries namely, Australia, United States, South Africa, United Kingdom and Canada. All the studies were conducted between March 2020 to August 2021. The studies included were crosssectional surveys, best practice recommendation, cover story and commentary.

Table 1 presents the characteristics of included studies in the present review.

Table 2 presents the changes in professional practice in terms of infection control measures, shift towards remote services, workplace related changes and prioritizing services.

\section{Discussion}

The present scoping review highlights the changes in audiology practice during COVID-19. Nine studies were included in the review, these included 5 cross-sectional surveys 2 cover stories, 1 recommendation and 1 commentary. The present review helps to understand the different changes in practice in audiology as a result of the COVID-19 pandemic and the restrictions. It also helps to identify the different challenges faced by audiologists in adapting to this change.

The different changes in audiology practice reported across the included studies in the could be categorized based on broad headings such as changes in infection control measures, shift to remote services, changes at workplace and prioritizing services. Infection control was always an important component in routine audiology practice due to direct and indirect contact with patients for prolonged duration and often for multiple visits It involves the use of probes, specula, headphones, and other instruments that are inserted in the ear. Further, most often the testing and management procedures take place in soundtreated closed setups. ${ }^{9,24}$ The onset of COVID-19 and the subsequent pandemic further highlighted and calibrated the need for more stringent infection control measures. Among the studies included, 5 studies recommended the implementation of strict infection control measures. These included use of face masks, face shields, gloves, social distancing, frequent cleaning of touch surface areas, reducing patient wait time and orienting and screening the patients before the actual appointment. ${ }^{16,17,20-22}$ As the pandemic continues to spread globally, it has become imperative that these infection control measures become a part of routine clinical practice.

Further, the COVID-19 pandemic hindered the standard routine audiology practice and led to exploration of the use of alternative options for service delivery. Tele practice acted as a quick alternative mode. Tele practices were identified to have the potential and longerterm opportunities since several years. ${ }^{25-27}$ However, in a study by Eikelboom and Swanepoe ${ }^{14}$ among audiologists from over 28 countries, reported that only $15.5 \%$ has experienced with tele-audiology. The reasons included were lack of infrastructure, cost, high case load at work limiting provision for tele practice. However, the 2019 COVID-19 pandemic brought a sudden shift to use of tele-audiology due to the unavailability of regular services. A shift of about $49-77 \%$ to remote services was reported in the included studies. ${ }^{16,18}$ This shift was a quick shift and led to a sudden uptake of remote services in audiology practice. Various modalities such as telephonic consultations, remote-services, video consultations, drop-off services, no-touch and low-touch options were used for providing services. ${ }^{8,18,21}$ This enabled in reducing the patient contact time to reduce the risk of infection ${ }^{8}$ as well as provision of services to remote areas. ${ }^{17}$

The COVID-19 pandemic also brough about changes at the workplace for several audiologists. $92 \%$ of the audiologists stopped working during pandemic ${ }^{20}$ in one study while in another only $5 \%$ audiologists provided regular care. ${ }^{19}$ In yet another study, $97 \%$ reported changes within their workplace with $76.4 \%$ reporting a drop in case load. ${ }^{17}$ In order to overcome with these changes, the audiologists adopted strategies such as shorter appointment timing, ${ }^{17}$ involving parents or teachers, ${ }^{19}$ using communication strategies. ${ }^{21}$ The pandemic also made audiologists prioritize patients based on diagnosis. Patients with complaints of sudden hearing loss, monitoring of ototoxicity, vestibular management got more priority. ${ }^{17,19,20,22}$

The findings of the review helped in identifying several challenges faced by audiologists while adapting to the changed audiological practice. These were categorized based on related to patients, related to remote services or specific practice related aspects. Majority of the challenges were related to adapting to remote services. As although tele audiology was available as a alterative means of service delivery, the pandemic and the unavailability of routine services forced many audiologists to shift quickly. These studies were conducted during the lockdown and at the start of the pandemic and as time passes, the audiologists might become more well-versed with using the remote 
Table 2

Changes in professional practice due to pandemic.

\begin{tabular}{|c|c|c|c|c|}
\hline Author ID & Infection control measures & Shift towards remote-services & Changes at workplace & Prioritizing services \\
\hline $\begin{array}{l}\text { Gunjawate } \\
\text { et al. }{ }^{17}\end{array}$ & $\begin{array}{l}\text { More than } 80 \% \text { used sanitizer, } \\
\text { handwash, masks, social distancing. } \\
39.3 \% \text { with standard procedures for } \\
\text { handwash. } \\
\text { Need for more standard operating } \\
\text { procedures for infection control. }\end{array}$ & $\begin{array}{l}77.3 \% \text { expressed interest to shift to } \\
\text { tele-practice }\end{array}$ & - & \\
\hline $\begin{array}{l}\text { Manchaiah } \\
\text { et al. }\end{array}$ & $\begin{array}{l}\text { More importance to infection control } \\
\text { measures }\end{array}$ & $\begin{array}{l}\text { Higher usage of remote services and } \\
\text { upgrading remote services }\end{array}$ & $\begin{array}{l}97 \% \text { changes at workplace, } 76.4 \% \\
\text { reduction in caseload, } 38.7 \% \text { reduced } \\
\text { work hours, } 25 \% \text { staff cuts or closure of } \\
\text { clinics, } \\
\text { - differences in counselling, service } \\
\text { delivery, triage, troubleshooting, } \\
\text { rehabilitation } \\
\text { - reduced appointment timings }\end{array}$ & $\begin{array}{l}\text {-Higher priority to audiological } \\
\text { support for device maintenance } \\
(53.4 \%) \text {, device adjustment }(53.3 \%) \text {, } \\
\text { cleaning and maintenance (48.4\%) } \\
\text {-Least priority to tinnitus } \\
\text { management }(72.1 \%) \text {, implant } \\
\text { support services }(71.4 \%) \\
\text {-Medium priority to emotional } \\
\text { support }(55.9 \%) \text { and psychosocial } \\
\text { support }(62.2 \%)\end{array}$ \\
\hline
\end{tabular}

used telephonic consultations, $20 \%$ used video consultation, $9 \%$ remote hearing screening services

Remote services most often used for treating management

Majority expressed interest to continue using tele-mode after lifting of restrictions $(83.7 \%)$.

$\begin{array}{cl}\text { Zaitoun } & 94.5 \% \text { avoided crowded places, } \\ \text { et al. }{ }^{21} & 93.9 \% \text { used sanitizer or washed } \\ & \text { hands regularly, } 88.4 \% \text { disinfected } \\ & \text { surfaces, } 85.4 \% \text { wore face masks, } \\ & \text { gloves. }\end{array}$

Use of tele-mode for imparting services to rural areas, $56.1 \%$ could reach to patients outside the conventional work practice redirected to other work $(9 \%)$

Majority appointments shifted to waitlist, varied alternatives used such as taking assistance from teachers of deaf, parents being asked to monitor children, doorstep drop of hearing aids, drop-off services, hybrid appointments

$82.9 \%$ minimized the waiting room time

90.9\% stopped working, $92.7 \%$

established new policies for dealing with pandemic, $63.4 \%$ received new training for managing cases,

Inform patients about new guidelines in advance, pre-screen all patients. Mail-in or drop-off services for devices. Use of communication strategies speak slowly, reduce ambient noise levels, rephrase and repeat, use of speech to text conversions/ communicator (transparent) facemask Limit personal interaction, especially with paediatric and geriatric population, 'drop off' service for hearing aid

Encourage use of Web and mobilebased applications for hearing care services like hearing assessment, hearing aid troubleshooting, finetuning, and counselling

Video-otoscopy, pure-tone audiometry, speech-in-quiet or a speech-in-noise audiometry test, objective measures of hearing, selfadministered screening or monitoring patients using smartphone or a tablet with an iOS or Android operating system.

Caregivers' role as facilitators in both
Audiology services based on no-touch (teleconsultation for digital proficiency assessment, hearing aid drop-off services, hearing aid troubleshooting, fine-tuning, counselling); low-touch service (screening, counter side service, self-testing kit for pure tone audiometry, speech-in-noise testing, digital otoscopy, hearing aids troubleshooting, counselling)
Most priority given to vestibular care

Priority areas -Assessment of sudden hearing loss (92.7\%) and post meningitis patients (85.4\%), followup of bilateral fail results in new-born hearing screening $(76.2 \%)$ and vestibular disorders with risk of fall (Meiners disease \& vestibular neuritis). Other areas such as hearing aids repair, earmold impression, replacement of lost aids, monitoring of ototoxicity.

Give priority to those with urgent needs.

High priority - Assessment of sudden sensorineural hearing loss, ototoxicity, idiopathic facial palsy, post meningitis followed by disorders like acute otitis media, acute mastoiditis, follow-up of new-born hearing screening, troubleshooting of hearing aid, and cochlear implant programming. 
Table 2 (continued)

\begin{tabular}{|c|c|c|c|c|}
\hline Author ID & Infection control measures & Shift towards remote-services & Changes at workplace & Prioritizing services \\
\hline & & $\begin{array}{l}\text { synchronous and asynchronous } \\
\text { modes. Recommended platforms for } \\
\text { tele practice include Koalys Confirm, } \\
\text { Koalys Consult, OtoPad, Shoebox Pro, } \\
\text { Kuduwave Plus \& Kuduwave Pro, } \\
\text { HearTest. } \\
\text { Use of tele-audiology for remote } \\
\text { training of future professionals } \\
\text { involved in the management of } \\
\text { hearing impairment. }\end{array}$ & & \\
\hline
\end{tabular}

service.

\section{Conclusion}

The present review explored the changes in audiology practice during COVID -19 and the challenges encountered by audiologists. This study will also help the audiologists and policymakers adjust to the audiology practice to cope with the ongoing pandemic. This study extends the directions for tele practice to become an integral part of routine practice.

\section{Declaration of competing interest}

There is no conflict of interest to disclose.

\section{References}

1 Madhav N, Oppenheim B, Gallivan M, Mulembakani P, Rubin E, Wolfe N. Pandemics: risks, impacts, and mitigation. In: The International Bank for Reconstruction and Development/the World BankDis. Control Priorities Improv. Heal. Reducing Poverty.. third ed. 2018 (Washington, DC).

2 Fernandez R, Lord H, Halcomb E, et al. Implications for COVID-19: a systematic review of nurses' experiences of working in acute care hospital settings during a respiratory pandemic. Int J Nurs Stud. 2020;111:103637.

3 Ives J, Greenfield S, Parry JM, et al. Healthcare workers' attitudes to working during pandemic influenza: a qualitative study. BMC Publ Health. 2009;9:56.

4 Lipsitch M, Swerdlow DL, Finelli L. Defining the Epidemiology of Covid-19 - Studies Needed. vol. 382. 2020:1194-1196. https://doi.org/10.1056/NEJMp2002125.

5 Moynihan R, Sanders S, Michaleff ZA, et al. Impact of COVID-19 pandemic on utilisation of healthcare services: a systematic review. BMJ Open. 2021;11, e045343.

6 Bowdle A, Munoz-Price L. Preventing infection of patients and healthcare workers should Be the new normal in the Era of novel coronavirus Epidemics. Anesthesiology. 2020;132:1292-1295.

7 Tan Z, Khoo DWS, Zeng LA, et al. Protecting health care workers in the front line: innovation in COVID-19 pandemic. J Glob Health. 2020:10.

8 Swanepoel DW, Hall JW. Making audiology work during COVID-19 and beyond. Hear J. 2020;73:20-24.

9 Kemp RJ, Roeser RJ. Infection control for audiologists. Semin Hear. 1998;19: 195-204.

10 The American Academy of Audiology. COVID-19 resources | the American academy of audiology. https://www.audiology.org/practice-resources/covid-19-resources/. Accessed August 2, 2021.
11 American Speech-Language-Hearing Association. Audiology service delivery considerations in health care during COVID-19. https://www.asha.org/aud/audiolo gy-service-delivery-considerations-in-health-care-during-coronavirus-covid-19/. Accessed August 2, 2021.

12 British Academy of Audiology. British academy of audiology response to covid-19: NHS England Essential activity for audiology. https://www.baaudiology.org/ind exphpnews/news-home/british-academy-audiology-response-covid-19-nhs-englandessential-activity-audiology/.

13 Indian Speech Language and Hearing Association. COVID-19 ISHA guidelines. htt ps://www.ishaindia.org.in/covid_19.html. Accessed August 2, 2021.

14 Eikelboom RH, Swanepoel DW. International survey of audiologists' attitudes toward telehealth. Am J Audiol. 2016;25:295-298.

15 Ravi R, Gunjawate DR, Yerraguntla K, Driscoll C. Knowledge and perceptions of teleaudiology among audiologists: a systematic review. J Audiol Otol. 2018;22: $120-127$.

16 Gunjawate DR, Ravi R, Yerraguntla K, Rajashekhar B, Ashwani V. Impact of coronavirus disease 2019 on professional practices of audiologists and speechlanguage pathologists in India: a knowledge, attitude and practices survey. Clin Epidemiol Glob Heal. 2021;9:110-115.

17 Manchaiah V, Eikelboom RH, Bennett RJ, Swanepoel DW. International survey of audiologists during the COVID-19 pandemic: effects on the workplace. Int $J$ Audiol. 2021:1-8.

18 Parmar B, Beukes E, Rajasingam S. The impact of COVID-19 on provision of UK audiology services \& on attitudes towards delivery of telehealth services. Int J Audiol. 2021:1-11

19 Saunders GH, Roughley A. Audiology in the time of COVID-19: practices and opinions of audiologists in the UK. Int J Audiol. 2021;60:255-262.

20 Zaitoun M, Alqudah S, Al Mohammad H. Audiology practice during COVID-19 crisis in Jordan and Arab countries. Int J Audiol. 2021:1-8.

21 Nalley C. Audiology amid COVID-19: reopening and infection control strategies. Hear J. 2020;73:28-31.

22 Saki N, Topsakal V, Amiri M, Jasim Al-shihaby W, Bayat A. The priority of audiological procedures during the COVID-19 pandemic. Med J Islam Repub Iran. 2021;35:770-772.

23 Thai-Van H, Bakhos D, Bouccara D, et al. Telemedicine in Audiology. Best Practice Recommendations from the French Society of Audiology (SFA) and the French Society of Otorhinolaryngology-Head and Neck Surgery (SFORL). Eur Ann Otorhinolaryngol Head Neck Dis; 2020.

24 Amlani A. Current trends and future needs for practices in audiological infection control. J Am Acad Audiol. 1999;10:151-159.

25 Muñoz K, Nagaraj NK, Nichols N. Applied tele-audiology research in clinical practice during the past decade: a scoping review. Int J Audiol.. 2021 https://doi.org/ 10.1080/14992027.2020.1817994, 23;60:S4-12.

26 Swanepoel DW, Hall JW. A systematic review of telehealth applications in audiology. Telemed J e Health. 2010;16:181-200.

27 Swanepoel DW. eHealth technologies enable more accessible hearing care. Semin Hear. 2020;41:133-140. 